\title{
LA RESPONSABILIDAD SOCIAL EMPRESARIAL. UNA ÓPTICA DESDE LOS STAKEHOLDERS
}

CORPORATE SOCIAL RESPONSIBILITY.

A PERSPECTIVE FROM THE STAKEHOLDERS

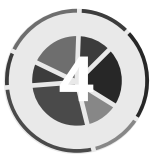

Recibido: 18/06/2017

Aceptado:03/07/2017

Johanna C. Armijos Cordero'

(johanna.armijos@ucuenca.edu.ec)

\section{Resumen}

El presente trabajo busca determinar el estado del arte sobre las diversas contribuciones a la Teoría de los Stakeholders en el marco de la Responsabilidad Social Empresarial, con el fin de determinar las variables que faciliten la medición de los elementos de valor que la empresa puede generar a través de sus stakeholders, dentro del contexto ecuatoriano. Se trata de una investigación que se enmarca en las áreas administrativa y financiera, de orden teórico, a través de la indagación bibliográfica y el análisis documental, que servirá como soporte para futuras investigaciones orientadas a establecer y medir las estructuras de las relaciones de las empresas con sus stakeholders, así como el valor que estos generan.

Palabras Clave: Responsabilidad Social Empresarial, stakeholders, Teoría del valor, competitividad.

\begin{abstract}
The purpose of this paper is to determine the state of the art into the various contributions to the Stakeholder Theory in the framework of Corporate Social Responsibility, in order to determine the variables that facilitate the measurement of the value elements that the companies can generate through their Stakeholders within the Ecuadorian context.

This is a framed research work in the areas: administrative and financial, with a theoretical order through bibliographic research and documentary analysis, which will serve as support for future researches aimed at establishing and measuring the structures of business relationships with their Stakeholders, as well as the value they generate.
\end{abstract}

Keywords: Corporate Social Responsibility, stakeholders, Value Theory, competitiveness

1 Carrera de Contabilidad y Auditoría. Universidad de Cuenca. ORCID 0000-0002-5597-1440 


\section{Introducción}

La Responsabilidad Social Empresarial (RSE) se refiere a la responsabilidad que tienen las empresas no solo de obtener beneficios económicos, sino también en el impacto social y ambiental de sus operaciones. Este término ha tenido gran discusión en los últimos tiempos gracias al desarrollo de las Tecnologías de Información y Comunicación, TIC's y la globalización. Aunque la RSE está teniendo gran aplicación en los países de primer mundo, sin embargo, en el Ecuador es un término apenas perceptible.

La RSE implica acciones que la empresa toma de forma íntegra y voluntaria, incorporando a sus actores en una espiral creciente de bien común, que tiene relación con el desarrollo de capital social y gestión del conocimiento, con el prestigio y la confianza, entre otros aspectos positivos para la empresa. De la RSE se ha derivado el término stakeholder, como el individuo o grupo de individuos que se ven o podrían verse afectados por las acciones u operaciones de la empresa, por lo que constituyen actores esenciales para el éxito de la empresa. La gestión de las relaciones con los stakeholders puede generar diversos tipos de valor que se podrían aprovechar para alcanzar competitividad, misma que no se alcanza de forma individual, sino es un desempeño superior que implica la interacción y colaboración de los otros.

\section{Revisión de la literatura}

\subsection{El bien común como bien trascendental del hombre}

El bien común va más allá del bienestar individual, ya que trasciende, perfecciona y realiza al ser humano; pero para alcanzarlo requiere del colectivo, de la interacción con la sociedad. Desde la perspectiva organizacional, implica que la empresa no solo tiene responsabilidades económicas, sino también sociales, yendo más allá de sus límites físicos. Su concepto trae consigo diversas perspectivas (Rivera \& Malaver, 2011, págs. 6-10):

- Instrumentales relacionadas con la existencia de circunstancias propicias a los miembros de una comunidad para alcanzar sus propósitos. 
- Distintivas referidas a las interrelaciones que se van plasmando con el interactuar entre los miembros de la comunidad.

- Agregadas que corresponden a la generación de bienes intrínsecos que pertenecen a la comunidad.

Sobre este tema, (Óp. Cit, pág. 11) es necesario destacar el rol social de la empresa, en donde el bien común actúa como mediador en la interrelación de esta con la sociedad. Es por ello que la responsabilidad social de las empresas es el bien común, "una empresa debe ser responsable con cada uno de los individuos y grupos con quienes tiene un vínculo directo, lo que permite que el bien común sea preservado" (Rivera \& Malaver, 2011)

\subsection{Evolución histórica de la Responsabilidad Social Empresarial}

No hay nada más social que emprender, ya que las empresas son creadas por personas, con personas y para personas. Aunque el beneficio económico es clave para que un negocio sea sostenible, es importante entender que este llega como resultado de un proceso; mientras mejor cimentado sea este proceso sobre una base social y moral, mejor será la competitividad del mismo, por lo que la noción de RSE implica una ruptura dicotómica entre Estado y empresa, así como entre sociedad y empresa, y entre empresa y ambiente; y ¿qué gana la empresa? pues su objetivo fundamental es maximizar su valor, en una negociación de ganar - ganar, construyendo así relaciones con sus stakeholders.

Aunque en la revolución agrícola o mercantil existieron manifestaciones éticas y responsables, en el siglo XIX hubo gran desigualdad, la industrialización trajo consigo el aumento de la pobreza, así como deplorables condiciones laborales, por lo que gran parte de la población dependía de la caridad, producto de los gestos de generosidad de las clases pudientes. No fue sino con la Revolución Industrial que la RSE empezó a generarse y extenderse (Rovira, 2016, pág. 28), cuando la población abandonó los campos y sus familias formando los núcleos urbanos, comenzando a experimentarse gran desigualdad social, surgiendo las primeras reflexiones respecto a la correspondencia entre empresa y sociedad; iniciaron las primeras manifestaciones sociales a través de colectivos que cues- 
tionaban la producción de productos perjudiciales para la salud, como el tabaco y el alcohol. (Op. cit. Pág. 29)

La evolución histórica de la RSE puede situarse en tres momentos (Correa \& Jaramillo, 2010, pág. 89):

- Una fase inicial que va desde el siglo XIX hasta inicios del siglo XX, con un nivel de RSE insípido en donde las instituciones solucionaban a su manera los diversos problemas sociales, esta fase se cristaliza en la primera mitad del siglo XX, en donde las organizaciones actúan voluntariamente, aunque de forma filantrópica. En el periodo posterior a la Segunda Guerra Mundial, hubo ya un concepto más moderno de la empresa, así como mayor consciencia de los problemas sociales como son: la pobreza, la exclusión y los desequilibrios económicos. En esta época, la OIT (Organización Internacional del Trabajo) delimita las pilastras de las corresponsabilidades que existen entre los actores económicos, políticos y sociales; a través de la Declaración de Filadelfia de 1944, que establecía temas trascendentales como la generación y las condiciones de empleo (Rovira, 2016, pág. 29).

- La segunda fase ocurre en la segunda mitad del siglo XX, cuando el modelo empresarial se fundamentaba preponderantemente en alcanzar beneficios económicos, resalta la postura de Milton Friedman, según lo cita (Mendez \& Peralta, 2014, pág. 632) quien indica que la única responsabilidad que tienen las empresas es optimizar sus recursos en búsqueda de beneficios para los accionistas. La sociedad comienza a despertar del letargo y los movimientos sociales reaccionaron ante prácticas reprobables o que financiaban economías éticamente censurables. En este contexto se inicia el debate respecto a las responsabilidades de las empresas, mismas que, ante la presión de las partes interesadas, tuvieron que dirigir su interés también hacia los aspectos sociales y ambientales de su accionar. Incluso el sector académico y el Estado comenzaron a dedicar su atención y a pronunciarse en este contexto. (Rovira, 2016, págs. 30-31)

- La tercera fase inicia en 1960 hasta la actualidad y se caracteriza por la fuerte integración entre Estado, Empresa y Sociedad. En 1971, la Declaración de Filadelfia concibió una propuesta formal sobre las responsabilidades sociales de las empresas, desde entonces han existido diversas iniciativas que incluso se transformaron en políticas sociales que buscaron 
un estado de bienestar o de "buen vivir". Como ejemplo está la Declaración de Estocolmo desarrollada por la ONU en el año 1972, con propuestas para preservación del medio ambiente. En 1987 el Informe Brundtland, con la definición de desarrollo sostenible relacionado a satisfacer las necesidades de las actuales generaciones sin comprometer la atención de las necesidades en el futuro. En los noventa, con la globalización, el constante examen del Estado de bienestar y la presión de grupos de interés, se produce una crisis de gobernabilidad que obligó a las empresas a transformar su forma de hacer negocios hacia prácticas más responsables, por lo que estas empiezan a formalizar sus acciones filantrópicas.

En 1997 la Organización para la Cooperación y el Desarrollo Económico (OCDE), creó el Global Reporting Iniciative (GRI) o Iniciativa para la Rendición de Cuentas Global, que determina pautas para presentar información sobre sostenibilidad; también en ese mismo año, el protocolo de Kyoto de la Convención Marco de las Naciones Unidas sobre el Cambio Climático y poco después, surgió el Instituto Ethos, como referente en RSE en Latinoamérica.

A partir de entonces y hasta la actualidad, han surgido un sinnúmero de instituciones y organismos que difunden RSE que han establecido normas, por ejemplo, las ISO 26000, las SQ 8000 (Social Accountability Standard 8000), las normas Adec Ethos, entre otras; entre los documentos sobre materia RSE están el Libro Verde Europeo, la Triple Cuenta de Resultados, etc. (Óp. cit. pág. 31-34)

\subsection{Concepto de RSE}

Aunque el concepto de RSE sigue en construcción, las empresas están comenzando a integrar la RSE dentro de su estrategia de negocio como un camino hacia la innovación y como un valor añadido tanto para el cliente, como para el accionista, (Óp. cit. pág. 35). Por otro lado, CERES ${ }^{2}$ (2008, págs. 7-11) señala algunas definiciones, recalcando la de la Unión Europea: "Integración voluntaria, por parte de las empresas, de las preocupaciones sociales y medio ambientales en sus operaciones comerciales y sus relaciones con sus interlocutores", cita a Forum Empresa: "Una visión de negocios que

2 Consorcio Ecuatoriano para la Responsabilidad Social 
integra armónicamente el respeto por los valores éticos, las personas, la comunidad y el medio ambiente", así como a Business in the Community- U.K.: "La RSE es entendida generalmente como la expresión del compromiso de una empresa con la sociedad y la aceptación de su rol en ella. Esto incluye el pleno cumplimiento de la ley y acciones adicionales para asegurar que la empresa no opera a sabiendas en detrimento de la sociedad. Para ser efectivo y visible, este compromiso debe estar respaldado por recursos, liderazgo y ser medible y medido".

Como se puede apreciar, la RSE tiene diversas perspectivas: está la deontológica que se fundamenta en lo axiológico a través de lo ético; la perspectiva utilitarista que usa la RSE como herramienta de gestión para la mejora de la competitividad y creación de valor para la compañía. Todo esto sumado a que existen diversos estudios que sostienen que las empresas que adoptan de manera efectiva la RSE como modelo de gestión son más rentables que aquellas que no. (Rovira, 2016, pág. 35)

En la actualidad, no hay ninguna normativa ni mecanismo que permita medir RSE, que sea suficientemente imparcial como para que, al ser aplicado indistintamente entre las empresas proporcione una medida del grado de responsabilidad social que estas tengan, la mayoría de los informes sobre RSE son elaborados por las mismas compañías, lo cual le resta objetividad. Incluso hay empresas que, aunque realizan acciones solidarias, sus directrices son contrapuestas a lo que las partes de interés requieren. (Óp. Cit. pág. 38-39).

\subsection{Estado de la RSE en Latinoamérica y en el Ecuador}

Los principales países que lideran la RSE a nivel mundial son: Suecia, Dinamarca, Finlandia, entre otros (Rovira, 2016). Latinoamérica se encuentra en un período de transición, sus avances en materia RSE no van a la par con los de primer mundo. Se pueden identificar tres grupos de países (Morán, Rodríguez, Torres, Aguilar, \& Villalta, 2016, págs. 23-24):

- Los de mayor incidencia como Brasil, Chile y México.

- Los de incidencia media entre los que se ubican Colombia, Argentina y los países centroamericanos. 
- Los de menor incidencia, en donde los avances en materia de RSE han sido mínimos, ya que su aplicación se presenta en casos aislados y en especial en empresas grandes, tal es el caso de Ecuador en donde además la presencia del Estado es sumamente frágil, y aunque la planificación ha estado enfocada hacia los Objetivos del Buen Vivir, no se ha logrado una verdadera institucionalización en beneficio de los stakeholders, debido a un sinnúmero de razones, entre las cuales, de manera general resaltan: la corrupción, la pérdida de confianza en el sistema, pobreza, desigualdad, entre otras.

En una investigación realizada por Torresano, M. (2012, págs. 25-35), las principales razones por las que en Ecuador no se ha implementado la RSE como estrategia en las empresas son: costos asociados, falta de formación del personal a cargo y falta de información de la RSE.

\subsection{Relación de la RSE con la innovación y con la competitividad}

Aunque las empresas consideran que adoptar RSE puede ayudarles a evitar ciertos riesgos económicos, minimiza las críticas públicas, mejora su posición en el mercado de consumo e incluso en el mercado bursátil; se debe tener en cuenta que adoptar RSE como modelo de gestión no es fácil, las empresas requieren alcanzar el equilibrio entre las dimensión social, medioambiental y económica; de no ser así, puede resultar un esfuerzo inútil o contraproducente para la reputación de la marca (ya que puede ser visto simplemente como un lavado de imagen) por lo que es necesario grandes esfuerzos de innovación en el plano social, lo cual resulta todavía un término genérico y poco definido. Innovar socialmente implica hacer lo mismo, pero de mejor forma; encontrando ideas nuevas que rompan paradigmas y que logren transformar la sociedad, añadiendo elementos responsables. (op. cit. pág 23-38).

"La innovación es el motor de la competitividad" (Peirano, 2014, pág. sn) y se trata de un producto humano que se adapta a condiciones específicas del entorno; por otro lado, la competencia global ya no se da entre empresas, sino entre sistemas complejos formados por cadenas de valor y redes (clústeres). En este escenario, las partes interesadas (stakeholders) son elementos clave para alcanzar la competitividad, y son por quienes, para quienes y con quienes las empresas deben trascender más allá del beneficio económico. 
La empresa no puede alcanzar competitividad por sí sola, Porter (1991) habla de lograr ventajas competitivas a través de un desempeño superior mismo que hay que sostenerlo. Requiere de un entorno que la favorezca, así como de un ambiente de institucionalidad y gobernabilidad por parte del Estado (Hernández, 2001, págs. 13-14). Por lo que la competitividad requiere de altas dosis de innovación, así como de la ayuda de los stakeholders. La llustración 1, muestra la clasificación de los stakeholders, en donde el consumidor es el stakeholder trascendental de las acciones de la empresa, teniendo como base, la sustentabilidad económica, social y ambiental. Así mismo, se esquematizan los diversos niveles de competitividad que se pueden alcanzar una vez que se han consolidado estrategias para gestionar cada uno de los grupos de interés.

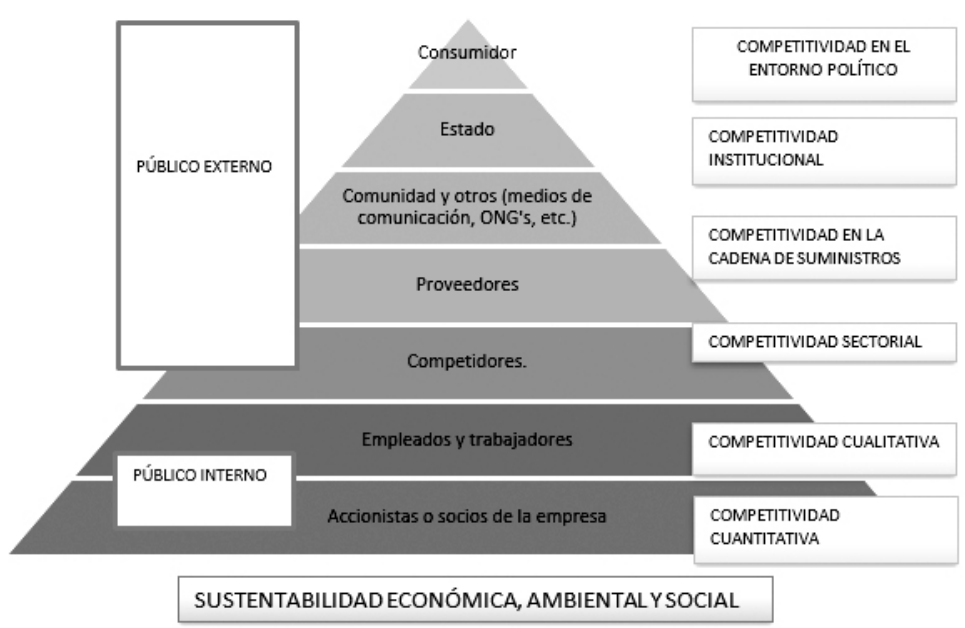

Ilustración 1: Pirámide de stakeholders

Fuente: (Peirano, 2014)

Elaboración propia

- La competitividad cuantitativa se alcanza volviendo atractiva la inversión hacia los accionistas, quienes proveen de capital a la empresa. Es responsabilidad de la empresa vigilar el mejor uso de dicho capital, optimizando el manejo de los activos tangibles, gestionando el riesgo financiero, así como los costos de producción, maximizando la rentabilidad. 
- La competitividad cualitativa tiene que ver con los activos intangibles, comprende el empoderamiento del recurso humano, la cultura organizacional así como la gestión del conocimiento.

- La competitividad sectorial está relacionada con el posicionamiento que la empresa tiene en el mercado, así como dentro de la industria.

- La competitividad de la cadena de suministros, dependerá de la integración vertical, así como de la gestión de los proveedores por parte de la empresa.

- La competitividad institucional tiene que ver con la conformación de redes (clústers), así como alianzas con demás organismos, tanto gubernamentales como no gubernamentales. Este tipo de competitividad permite mejorar el capital social (se va construyendo a través de las interrelaciones entre las personas dentro de una organización, con otras organizaciones y entre organizaciones entre sí). Los clústeres son "masas críticas de empresas que actúan en industrias relacionadas con asiento en una región". Porter (1998, pág. 32), define a los clústeres como: "agrupaciones mayores de instituciones que se acumulan, basados en integraciones tanto horizontales (cadenas) como verticales (tipos de cadenas diferentes que se relacionan entre ellas para mejorar la competitividad de sus componentes, apoyándose, intercambiando ideas y creando centros de innovación)", permitiendo a las empresas lograr ventajas competitivas que les permitan acceder a mercados internacionales, mejorando la cadena de suministro y hacer frente a las amenazas del entorno (Restrepo, 2004, pág. 12).

- La competitividad en el entorno político de mayor trascendencia en la pirámide, se alcanza cuando, a través de la gestión de los stakeholders, se ha logrado la creación de valor compartido, así como sostenibilidad y sustentabilidad para la empresa y para todos los grupos de interés en una negociación de ganar - ganar. Es necesario que la creación de valor en la empresa sea de forma compartida, creando también valor para la sociedad y para los grupos de interés, asegurando beneficios también para su entorno en el largo plazo, aprovechando las interrelaciones recíprocas entre el bienestar de la comunidad y el de la empresa. La innovación es vital al incorporar nuevas formas de uso de herramientas existentes que consideren esta interdependencia (Méndez \& Peralta 2014, pág. 627).

El consumidor es hacia quien apuntan todas las estrategias de RSE (y las de competitividad), pero como se puede apreciar, implica un recorrido estratégico 
con actores clave, cuyos beneficios son, a nivel interno: la mejora en el empoderamiento de los empleados, reduciendo costos, mejorando el clima laboral y la comunicación interna, todo esto se ve traducido en mejor productividad y calidad; y, a nivel externo la fidelización de clientes, mejora la imagen y reputación de la empresa y de la marca, permitiendo acceder a nuevos segmentos de mercado.

Mendez \& Peralta, $(2014,628)$ citan a Carlos Llano-Cifuentes, quien indica que los nuevos fines de las empresas deben ser: “a) generación de valor económico agregado, b) servicio a la comunidad, c) desarrollo de las personas que integran la empresa y d) capacidad de continuidad". En estos fines, el deber de los stakeholders es aportar al bien común de la empresa, si alguno de estos actores desconoce su responsabilidad sobre el bien común, esto podría afectar al bien común de la sociedad en sí (Rivera \& Malaver, 2011, pág. 11).

\subsection{Teoría de los stakeholders}

De la RSE se ha desprendido la Teoría de los Stakeholders, en este sentido existen numerosas investigaciones, de las cuales se destacan las siguientes:

- La propuesta de González (2010, págs. 39-57), enfatiza sobre que, con el desarrollo de las TIC's, se dan nuevas interrelaciones entre las empresas y sus stakeholders.

- Por otro lado, González (2007, págs. 205 - 227) subraya que la gestión de los stakeholders facilita el llevar a la práctica la ética.

- Resulta interesante lo señalado por Toro (2006), sobre que la aplicación de RSE debe conjugar las estrategias social y económica, permitiendo rentabilidad financiera y agregando valor.

- A lo anteriormente mencionado, Acuña (2012) añade que la atención a la gestión de stakeholders favorece la sostenibilidad y la dirección estratégica de la empresa, realizando un análisis comparativo entre las herramientas disponibles para la gestión de stakeholders.

- Finalmente, Lorca (2003, págs. 48 - 54), contribuye a la Teoría de los Stakeholders, definiendo las relaciones que hacen que las partes interesadas agreguen valor a la empresa. 
Rivera \& Malaver, (2011, pág.10) clasifican las siguientes fases de desarrollo del concepto de stakeholders:

- Primera fase: evidencia la necesidad de relacionar negocios y sociedad, algunos de sus autores fueron: Berle y Means, Boulding, Selekman, Pierson, entre otros.

- Segunda fase: con un enfoque en negocios - sociedad y responsabilidad social corporativa, entre sus autores están: Adams, Austin, Boulding, Elbing.

- Tercera fase: hay una sensibilización social corporativa, en donde están autores como Frederick, con quien el enfoque se direccionó hacia la responsabilidad social corporativa.

Sin embargo, fue Freeman, (Óp. Cit. pág. 20), quien en 1984 popularizó el término stakeholder, como "cualquier grupo o individuo que puede afectar o ser afectado por la consecución de los objetivos de la empresa", a lo que (Baro, 2011, pág. 140), añade que el stakeholder "provee recursos críticos o activos a la organización y los arriesga a cambio de un beneficio".

Donalson citado por Rivera y Malaver (2011, pág. 26), sostiene que "stakeholders son personas o grupos con intereses legítimos en los aspectos procedimentales y/o sustantivos de la corporación", a lo que fueron aportando luego autores como Mitchell, Agle y Wood (1997, págs. 865-873), quienes establecieron un mecanismo para identificar los stakeholders según tres atributos básicos ante los cuales, Baro, (2011, 140-141) propone que la gestión de vincular con los stakeholders: "contribuye a la gestión del capital social, fuente de ventajas competitivas" y cita a Rawlins sobre su modelo sistemático e integral de gestión de vínculos con los stakeholders en cuatro pasos:

- Identificación de stakeholders, que consiste en elaborar un listado exhaustivo de stakeholders, estableciendo sus vínculos según sean:

- Vínculos posibilitadores: aquellos que facilitan el funcionamiento de la empresa.

- Vínculos funcionales: aquellos que construyen, por un lado, el input y por otro el output de la empresa. 
- Vínculos normativos: aquellos con quienes comparten realidades o valores.

○ Vínculos difusos: cuya contribución no está bien definida, como los líderes comunitarios, ONG's ${ }^{3}$.

- Priorización por atributo, Mitchel, Agle, \& Wood (1997, págs. 865-873), exponen un Modelo de Atributos y Prominencia, que clasifica a los stakeholders en función de tres atributos:

- Poder: este atributo tienen los stakeholders que pueden influir en las decisiones organizacionales.

- Legitimidad: este atributo poseen aquellas partes interesadas que pueden reclamar un determinado proceder a la organización.

- Urgencia: ocurren bajo dos situaciones, cuando los requerimientos de los stakeholders necesitan atención inmediata y cuando estas resultan críticas.

Los stakeholders que poseen un solo atributo se llaman latentes, debido a su baja prominencia; aquellos que posean dos, presentan prominencia media y se llaman expectantes y los que posean los tres atributos a la vez tienen una prominencia alta y son denominados definitivos.

- Priorización por situación, Baro, $(2011,147)$ cita a Hallahan, clasificando a los stakeholders según el nivel de conocimiento que posean sobre la organización, así como según su grado de implicación, respecto a lo cual, se pueden clasificar en:

- Inactivos: con un nivel de conocimiento e implicación bajo.

- Atentos: los que, aunque están altamente involucrados, su nivel de conocimiento es bajo.

- Informados: quienes poseen alto grado de conocimiento, sin embargo, bajo grado de involucramiento.

- Activos: son quienes están muy involucrados y a la vez tienen elevado grado de conocimiento.

3 Organización No Gubernamental 


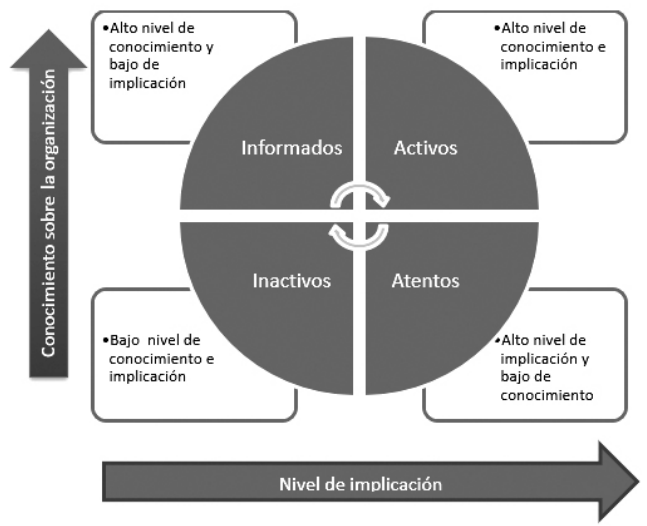

Ilustración 2: Priorización por situación

Fuente: Baro (2011, pág. 147)

Elaborado por: La autora

Si no posee ningún grado de conocimiento ni de involucramiento, no puede ser denominado stakeholder. En este mismo sentido el autor otorga una jerarquía a cada clasificación, según su grado de urgencia: en primer lugar, los Activos, seguido de los Atentos, luego los Informados y, por último, los Inactivos.

\section{- Priorización por estrategia comunicacional}

Solo aquellos stakeholders que tienen mayor jerarquía, es decir, los más atentos y activos, pueden llamarse públicos, "un público es un stakeholder que, por sus atributos, hemos identificado como uno con alta jerarquía y, lo que es más importante, al cual hemos decidido destinar acciones comunicacionales que lo mantengan, o lo transformen en atento o activo", (Óp. cit. pág. 149)

En esta etapa se pretende desarrollar estrategias comunicacionales que permitan transformar o mantener a los stakeholders lo más atentos o activos posibles, volviéndolos públicos. 


\subsection{Situación de los stakeholders en Ecuador}

En Ecuador los grupos de interés ejercen presión para que las empresas definan una estrategia de RSE, en el año 2008 los principales stakeholders en exigir prácticas responsables eran: clientes, comunidades y sociedad (Torresano, 2012, pág. 30).

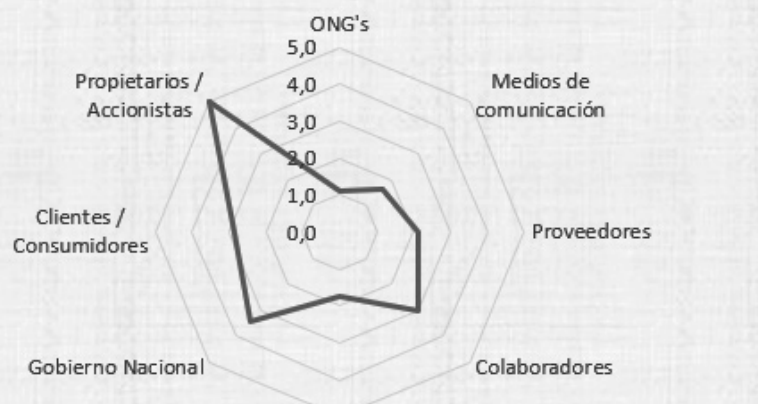

Ilustración 3: Principales stakeholders que presionan por estrategias de RSE

Fuente: Torresano (2012, pág. 30)

Elaborado por: La autora

En el año 2012, como se puede apreciar en la llustración 3, los principales stakeholders que ejercen presión para que se apliquen estrategias de RS son los propietarios y accionistas, seguido del gobierno y, en tercer lugar, los colabo-

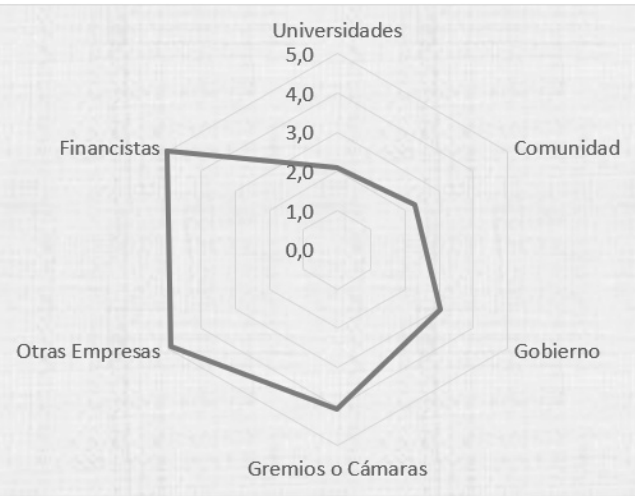

Ilustración 4: Nivel de relacionamiento de la empresa con sus stakeholders

Fuente: Torresano (2012, pág. 33)

Elaborado por: La autora 
radores. Respecto al año 2008, se puede advertir que ha existido un importante cambio, pasando de ser los grupos externos a grupos internos los stakeholders que exigen prácticas responsables por parte de las empresas.

Las empresas en Ecuador se relacionan mejor con los financistas, con otras empresas y con los gremios a los cuales pertenecen, ver llustración 4 .

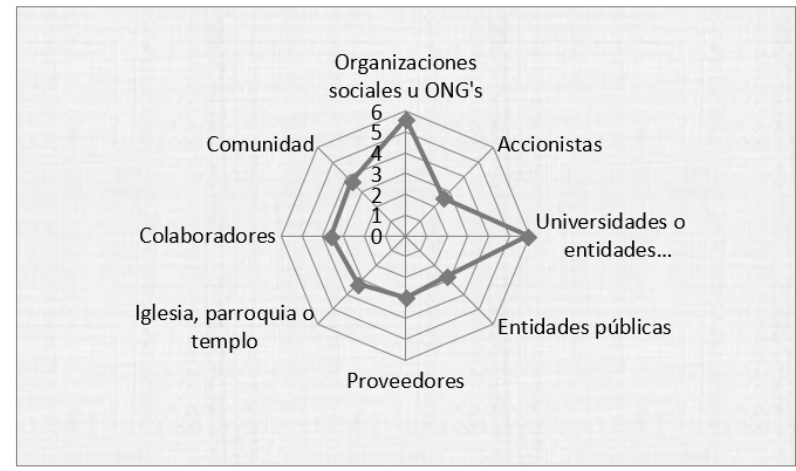

Ilustración 5: Temas de RSE de mayor interés para las empresas

Fuente: Torresano, M. (2012, págs. 37-38)

Elaborado por: La autora

Lo anotado indica que las estrategias de RSE podrían implementarse mejor si se desarrollan a la par estrategias de competitividad sectorial, mediante políticas públicas que promuevan, por ejemplo, la conformación de clústeres.

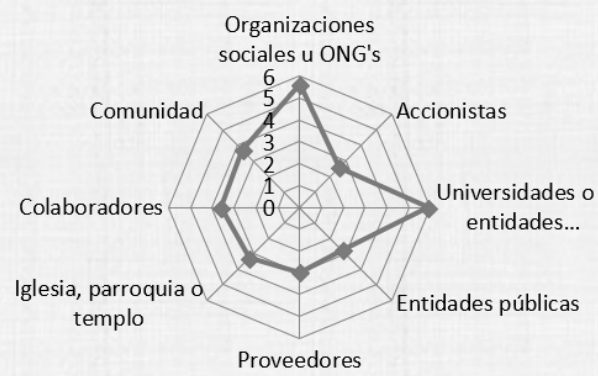

Ilustración 6: Principales beneficiarios de la RSE de las empresas

Fuente: Torresano, M. (2012, pág. 32)

Elaborado por: La autora 
Como se puede apreciar en la llustración 5 , los temas que más interesan a las empresas en materia de RSE son los Derechos Humanos, seguidos de las prácticas laborales y la responsabilidad del producto.

La llustración 6, muestra los principales beneficiarios de las acciones de RSE de las empresas en el año 2012 en el Ecuador, lo cual implica que las organizaciones sociales y las universidades y entidades educativas han sido los mayores beneficiarios de las prácticas de RSE, esto conlleva a ratificar lo señalado por diversos autores, sobre que en el Ecuador las prácticas de RSE son aisladas y no contribuyen al éxito empresarial, además no se encuentran articuladas de manera estratégica hacia el logro de competitividad.

Adicionalmente, como sostiene Rovira (2016, págs. 37-39), hay una gran diferencia entre lo que las empresas dicen que hacen y lo que realmente llevan a la práctica. Por todo lo señalado, mientras no se encuentren cimentados los elementos de RSE en el quehacer diario de la empresa aunando la visión social con la gestión empresarial, cualquier acción que la empresa desarrolle en materia de RSE puede resultar inútil o incluso, hasta contraproducente.

\subsection{Creación de valor a través de la RSE}

Desde la perspectiva de la teoría neoclásica, el valor económico se explica como la diferencia entre el precio que se paga por un bien o servicio y su costo de producirlo, la creación de valor económico está relacionada principalmente con dos aspectos: la satisfacción del consumidor y la eficiencia en cuanto al uso de recursos para producir. Desde otra perspectiva, se trata de "un sistema analítico concebido para descomponer las distintas funciones de una empresa y examinar el coste que tienen. Su objetivo es asignar los recursos a los largo de la cadena de la forma más eficaz posible" (50minutos.es, sf) logrando así ventajas comparativas a través de estrategias de precio o de diferenciación.

Pero, ¿quién se lleva este valor? O más bien ¿Para quién la empresa debe generar este valor?. Pues hay quienes defienden que, una vez pagados salarios e insumos que el mercado y la ley definen, se lo debería llevar el propietario de la empresa. Pero ¿qué ocurre si baja el precio?, parte de este valor se lo estará llevando el clien- 
te, ¿qué ocurre si el precio disminuyó porque bajó el salario de sus empleados?, pues el valor para los empleados se redujo lo cual significa que el valor generado pasó a otra parte interesada. En este sentido, es necesario identificar que existe una gran diferencia entre que la empresa genere valor y quién se lleva dicho valor, respecto a lo cual ha existido amplia discusión, desde Freeman citado por Argadoña (2011, pág. 3), quien sostiene que la empresa debe ser gestionada para sus Stakeholders y no sólo para sus accionistas, luego Post, Preston y Sach, (Óp. Cit. págs. 3-5) añaden que el valor creado debe ser el mayor posible, hay que considerar que además hay otras partes interesadas que, aunque no participan directamente en este proceso de creación de valor por parte de las empresas, se ven afectadas directa o indirectamente de este proceso, como por ejemplo, la comunidad y el ambiente.

Hay un tipo de valor que trasciende al valor económico (que se podría catalogar como extrínseco) y que resulta invisible, en un sentido aún más amplio que lo tratado hasta el momento, y se refiere a lo que cada uno siente y percibe como valor o valioso (un resultado intrínseco): la satisfacción que tiene el empleado por cumplir su trabajo y que lo reconozcan, el agrado que tiene el consumidor cuando adquiere un producto que percibe de calidad y que en su producción no se haya afectado a la naturaleza o a la sociedad (Vives \& Peinado - Vara, 2011, págs. 5682). Todos estos valores, ya sean económicos o no, no cabe duda que se generan socialmente, pues requieren interacción social, por ejemplo: clientes - empresa, proveedores - empresa, empleados - administradores, administradores - accionistas, etc. De esa interacción se generan valores que en algunos casos se transfieren, como cuando el cliente paga el precio del producto, y en otros casos estos valores se proporcionan cada uno a sí mismo, como la satisfacción del empleado por su trabajo, (Óp. cit. Págs. 1-5)

Lo interesante que diferencia al valor económico del no económico radica en que, del valor económico se pueden apropiar los demás y se tiene que renunciar a uno para alcanzar otro; esto no ocurre con el valor no económico que, al no poder ser apropiado por otros, perdura en cada uno en el tiempo: los aprendizajes, las experiencias o los valores morales.

Al hablar de que la empresa debe maximizar el valor para sus stakeholders, es importante considerar, tanto el valor económico como el no económico; (Óp. Cit. Págs. 8-9), propone seis tipos de valores: 
1. Valores extrínsecos económicos, que son los llamados valores económicos propiamente dichos.

2. Valores extrínsecos inmateriales, como, por ejemplo: el reconocimiento, la formación, etc., que complementan los valores económicos.

3. Valores intrínsecos psicológicos, se forman en cada persona, como la satisfacción por el trabajo realizado.

4. Valores intrínsecos de aprendizaje adquirido por el agente y no en la empresa, aunque no forma parte del valor económico creado, pero puede contribuir en un futuro a la creación de valor económico.

5. Valores trascendentes están en el campo de la ética, aprendizajes evaluativos que se generan en el agente producto de sus decisiones y las consecuencias que han tenido las mismas, condicionarán las decisiones dentro de las cuales se enmarcarán todos los demás valores.

6. Valores que son externalidades, positivas o negativas, en agentes distintos a los que se trata la relación, pero tienen un impacto sobre esta.

\subsection{La Cadena de Valor}

Para Porter (1991), una organización es una cadena que crea valor entre sus stakeholders, es en esta cadena de valor en donde se pueden obtener ventajas competitivas, a través del desarrollo de estrategias en cada uno de sus componentes, los mismos que son:
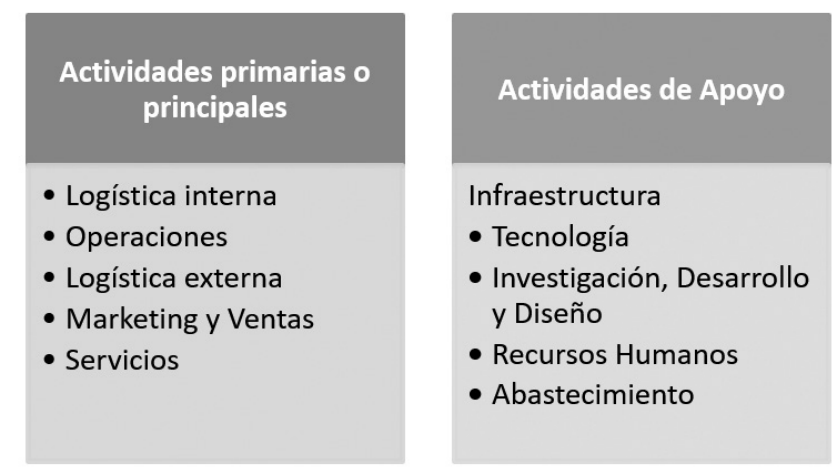

Ilustración 7: Componentes de la Cadena de Valor

Fuente: Porter, M. (1991)

Elaborado por: La autora 
En la llustración 7, se aprecian los componentes de la cadena de valor, en donde las actividades primarias están relacionadas a la fabricación, comercialización y servicio postventa de la propuesta de valor de la empresa, por otro lado, las actividades de apoyo sostienen a las actividades primarias, permitiéndoles fluir. El margen resultará en la diferencia entre el valor total generado por la empresa y el costo total de generación de ese valor. En toda esta cadena de valor intervienen los stakeholders, mismos que, al generar valor con sus acciones, también son partícipes y demandantes de este valor generado. La gestión estratégica de la cadena de valor genera ventaja competitiva, sobre todo al ser combinada con su economía y comparada con su competencia, a la vez que, buscará generar valor satisfaciendo las necesidades del comprador y obligándose así a desarrollar estrategias de diferenciación, todo esto constituye una poderosa herramienta estratégica de ventaja competitiva.

\section{Materiales y métodos}

Se aplica la revisión de la literatura científica existente en relación a la Responsabilidad Social Empresarial y la Teoría de los Stakeholders, combinando métodos como el análisis y síntesis, separando a la teoría de sus partes y luego integrando aquellas que tienen mayor relevancia; así también el método inductivo, a través de la búsqueda de elementos que explican las relaciones de las empresas con sus stakeholders; y deductivo, mediante la búsqueda de concreciones de esta teoría, por último, la analogía sirve como instrumento para la determinar el estado de la gestión en RSE en el Ecuador, con relación a la Teoría de los Stakeholders.

\section{Discusión y resultados}

La Responsabilidad Social Empresarial es una visión empresarial que está teniendo cada vez más acogida y discusión, su aplicación está más arraigada en los países del primer mundo, sin embargo, en el Ecuador se lo hace de forma aislada, esto se evidencia en especial porque no hay concordancia entre los temas de interés de las empresas que afirman gestionar con RSE y los beneficiarios de las mismas; de igual manera ocurre con quienes presionan a las empresas por prácticas respon- 
sables y con quienes en efecto dichas empresas ponen énfasis en la construcción de relaciones. De lo que no hay duda, es que la gestión en RSE resulta un tema clave para alcanzar beneficios mutuos para todos los actores relacionados con las empresas, logrando así un desempeño superior que está ligado a la competitividad.

La Teoría de los Stakeholders otorga pautas de cómo, mediante la gestión de relaciones con los stakeholders, se pueden generar diversos tipos de valor, pero hay un tipo de valor que trasciende al valor económico extrínseco y que es invisible y representa el valor percibido, es decir, un elemento intrínseco y que se considera determinante en la obtención y mantenimiento del valor económico en el corto, mediano y largo plazo. Este valor puede ser interno de la empresa, como por ejemplo el empoderamiento de los empleados, o la mejora del clima laboral que permite desarrollar las condiciones de aprendizaje, especialización y gestión del conocimiento; con lo cual, a la larga, se reducen costos. A nivel externo, el valor tiene relación, por ejemplo, con la mejora en la reputación de la empresa, facilitando así el acceso a financiamiento, así como la aplicación de estrategias de marketing.

Los tipos de valor intrínsecos señalados adquieren mayor relevancia cuando el objetivo es alcanzar sostenibilidad y sustentabilidad, que resultan claves para la supervivencia de las empresas en el mediano y largo plazo. Lo anteriormente mencionado lleva a pensar que para las empresas es de vital importancia, además de buscar valor económico, el promover el desarrollo del grupo humano que la conforma, edificar relaciones que perduren con la comunidad y con el ambiente, con los proveedores, con los clientes, e incluso con la competencia, potenciando así su sostenibilidad en el tiempo.

En la llustración 8 se pueden observar los diversos tipos de valor, tanto extrínseco como intrínseco y trascendentales que pueden aportar los stakeholders a través de la cadena de valor, todos estos valores se pueden alcanzar a través de la construcción de relaciones mediante la gestión de los stakeholders, la mayoría de los cuales no son extrínsecos económicos y resultan cruciales para alcanzar ventajas competitivas para la empresa, estos requieren de cierto tiempo para conseguirlos, por lo que necesitan que las empresas realicen una gestión estratégica de RSE, poniendo énfasis en alcanzar estos valores en el largo plazo por sobre los beneficios que puedan tener en el corto plazo. 


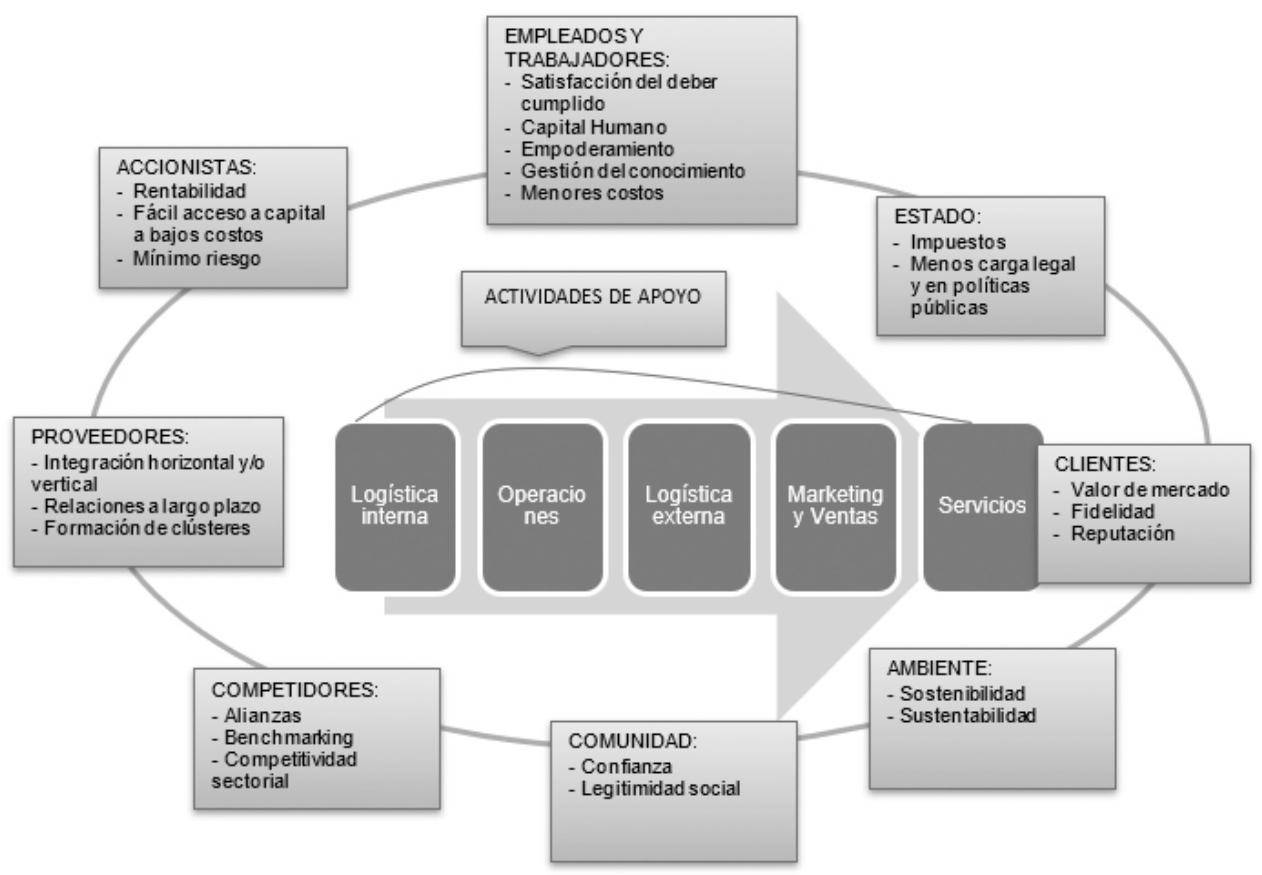

Ilustración 8: Valor que aportan los stakeholders en la Cadena de Valor

Elaboración propia

\section{Conclusiones y recomendaciones}

La gestión en RSE es un tema prioritario para los países y para las empresas para alcanzar competitividad y asegurar sostenibilidad y sustentabilidad, atendiendo no únicamente los objetivos económicos, sino también los sociales.

La gestión en RSE implica una gestión social, ninguna de las estrategias de RSE pueden ser aplicadas por la empresa de forma individual, requieren interacción con otros actores, mediante la construcción de relaciones beneficiosas para ambas partes, por lo que la mentalidad de todos los actores debe cambiar, es necesario abrir mecanismos de diálogo, de asociación y de integración, así como políticas públicas que faciliten y promuevan la concertación. 
Los stakeholders constituyen actores fundamentales en la cadena de valor, la construcción de relaciones con cada uno de ellos puede llevar a las empresas a la generación de ventajas competitivas que permitan mejorar los sistemas económicos en su conjunto.

En el Ecuador, la aplicación de RSE es muy débil, uno de los grandes obstáculos es el desconocimiento de los temas relacionados con la RSE, en este sentido la academia se vuelve un actor fundamental para minimizar esta dificultad en el sector empresarial, ya que existe gran confianza del mismo con las universidades e institutos de educación. En este mismo sentido, el sector académico debe aprovechar esta relación para desarrollar sus funciones de docencia, de investigación y de vinculación con la colectividad.

Es muy importante para todos los actores económicos y sociales, gestionar los elementos de valor que pueden aportar los distintos stakeholders para alcanzar una competitividad sustentable y sostenible, no únicamente en las empresas sino de manera sistémica, a nivel de país, y por qué no decirlo, a nivel de región.

Los mecanismos de medición que existen al momento en respecto a RSE no se adecúan a la realidad de las empresas, es necesario que la academia establezca herramientas de medida de RSE que sean imparciales, pertinentes, confiables y accesibles. De esta manera se asegura transparencia sobre la información que brindan las empresas sobre su impacto en las dimensiones económica, social y ambiental.

Resulta vital en las empresas la gestión estratégica con RSE teniendo en cuenta la gestión del valor, para que las empresas logren un desempeño superior. Al igual que con la RSE, resta por construir modelos de medición de dicho valor, ya que es un factor crítico de éxito para las empresas y para los sistemas económicos.

De todos los tipos de valor que puede obtener una empresa, los intrínsecos son los más numerosos y deben ser considerados de gran importancia para el logro de los objetivos de las empresas ya que son la base de todos los procesos en su cadena de valor. Una ventaja relevante de los valores intrínsecos y trascendentales es que no se extinguen, como ocurre con el valor económico, del que cada stakeholder toma una parte. 


\section{Referencias bibliográficas}

- 50minutos.es. (sf). La Cadena de Valor de Michael Porter. Identifique y optimice su ventaja competitiva. Economía y Empresa.

- Acuña, A. (2012). La Gestión de los Stakeholders. Análisis de los diferentes modelos. Universidad Nacional del Sur, Encuentro Regional Zona Sur Adenag.

- Argadoña, A. (2011). La Teoría de los Stakeholders y la Creación de Valor. Business School - Universidad de Navarra, 1-13.

- Baro, M. (2011). Jerarquización de stakeholders para la construcción del capital social de las organizaciones. Mediaciones Sociales(9), 135-162.

- CERES. (2008). El ABC de la Responsabilidad Social Empresarial RSE. Consorcio Ecuatoriano para la Responsabilidad Social Empresarial. Quito: Mantis Comunicación.

- CERES. (2018). Consorcio Ecuatoriano para la Responsabilidad Social. Recuperado el 15 de 04 de 2018, de http://www.redceres.com/

- Correa, G., \& Jaramillo, J. (2010). Evolución histórica de los conceptos de Responsabilidad Social Empresarial y Balance Social. Semestre Económico, 87-102.

- Cuesta, M., Pardo, E., \& Paredes, J. (2013). Identificación de indicadores relevantes del desempeño RSE mediante la utilización de técnicas multicriterio. Innovar, 75-88.

- Diario La Hora. (02 de 05 de 2017). Libertades y derechos en Ecuador preocupan a la ONU. Diario La Hora.

- Diario La Hora. (17 de 04 de 2017). Un gobierno que inspire confianza . Diario La Hora.

- Estévez,A. (2005). Reflexiones teóricas sobre la corrupción: sus dimensiones política, económica y social. (U. d. Zulia, Ed.) Revista Venezolana de Gerencia, 10(29), 43-85.

- González, C. (2010). E-Stakeholders: Una aplicacion de la teoria de los Stakeholder a Los Negocios Electrónicos. Estudios Gerenciales, 39-57.

- González, E. (2007). La teoría de los stakeholders. Un puente para el desarrollo práctico de la ética empresarial y la responsabilidad social corporativa. Veritas, 205 - 224. 
- Guillermo, C. J. (2010). Evolución histórica de los conceptos de Responsabilidad Social Empresarial y Balance Social . Semestre Económico, 87-102.

- Habermas, J. (2003). La ética del discurso y la cuestión de la verdad. Escuela de Filosofía Universidad de ARCIS.

- Hernández, R. (noviembre de 2001). Elementos de competitividad sistémica de las pequeñas y medianas empresas (PYME) del Istmo Centroamericano. (C. ECLAC, Ed.) Estudios y Perspectivas, 1-41.

- Jaramillo, I. (julio - diciembre de 2015). Reflexiones de la responsabilidad social empresarial desde su dimensión laboral. Espiga(30), 9-21.

- Lorca, P. (2003). La creación de valor en la empresa y los "stakeholders". Harvard-Deusto Finanzas \& Contabilidad,, 48 - 54.

- Lorenzo, C., Camio, M., \& Alzola, M. (2000). El capital social organizacional: Patrimonio intangible del modelo comunicacional. Cuaderno de Administración, 119-135.

- Mendez, J., \& Peralta, D. (2014). Reflexiones respecto a la responsabilidad social empresarial y la creación de valor económico desde la perspectiva de los proveedores. Cuaderno de Contabilidad, 625-645.

- Méndez, J., \& Peralta, D. (2014). Reflexiones respecto a la RSE y creación de valor económico desde la perspectiva de los stakeholers. (U. Javeriana, Ed.) Cuadernos de Contabilidad, 624-645.

- Mesa-Gilbert, C. (2005). La lucha contra la corrupción. En B. Klicksberg, La Agenda ëtica pendiente de América Latina (págs. 213-218). Buenos Aires: Fondo de Cultura Económica.

- Mitchel, R., Agle, B., \& Wood, D. (octubre de 1997). TOWARD A THEORY OF STAKEHOLDER IDENTIFICATION AND SALIENCE: DEFINING THE PRINCIPLE OF WHO AND WHAT REALLY COUNTS. Academy of Management, 853-886.

- Morán, C., Rodríguez, L., Torres, M., Aguilar, A., \& Villalta, M. (junio de 2016). Stakeholders, Responsabilidad Social en Ecuador. Revista Científica y Tecnológica UPSE, III(2), 21-30.

- Osterwalder, A. (24 de 10 de 2012). Mejora Competitiva. Recuperado el 29 de 01 de 2018, de https://www.mejoracompetitiva.es/2012/10/alexosterwalder/ 
- Peirano, C. (06 de 2014). La Pirámide de la Competitividad y su Aplicación al Análisis Competitivo del Sector Forestal. Visión del Futuro, sn.

- Peláez, J., \& García, M. (2014). Responsabilidad Social Empresarial. (U. Libre, Ed.) Entramado, 10(2), 90-111.

- Peña, D., \& Serra, A. (julio - septiembre de 2013). La práctica de la responsabilidad social empresarial., Estudio de caso en el sector turístico. (U. N. Colombia, Ed.) Innovar, 23(49), 101-113.

- Peña, D., \& Sierra, A. (noviembre de 2012). Responsabilidad Social Empresarial en el Sector Turístico. Estudio de caso en empresa de alojamiento de la ciudad de Santa Marta, Colombia. (C. d. Turísticos, Ed.) Estudios y Perspectivas en Turismo, 1456-1480.

- Peña, D., Serra, A., \& Cardona, J. (julio - didciembre de 2017). Perfil característico y responsabilidad social empresarial del sector hotelero de la región caribe colombiana. Pensamiento y gestión(43), 128-149.

- Peña, D., Serra, A., \& Ramón, J. (abril-junio de 2017). Factores determinantes del conocimiento de la Responsabilidad Social Empresarial en el sector hotelero del Caribe colombiano. Revista Ibero-Americana de Estrategia, 16(2), 104-124.

- Porter, M. (1991). Ventaja Competitiva. Creación y Sostenimiento de un Desempeño Superior. México DF: CECSA.

- Porter, M. (1998). The Dawn of the E-Lance Economy. Academia, 130-145.

- Prats, J. (2005). Ética para el buen oficio político. En B. Kliksberg, La agenda ética pendiente de América Latina (págs. 167-212). Buenos Aires: Fondo de Cultura Económica de América Latina.

- Restrepo, F. (2004). Interpretando a Porter. Rosario - Colombia: Centro Editorial - Universidad de Rosario.

- Rivera, H., \& Malaver, M. (2011). La organización: los stakeholders y la responsabilidad social. Bogotá: Universidad de Rosario .

- Rovira, C. (2016). Responsabilidad Social Competitiva. Barcelona: Empresa Activa.

- Sánchez, M., \& Gallardo, D. (2013). El papel del Tercer Sector ante la Responsabilidad Social Empresarial, Un análisis cualitativo de la realidad extremeña. CIRIEC-ESPAÑA, 77, 59-86. 
- Sepúlveda, J., Ordóñez, F., \& Prada, C. (enero de 2014). Perfil de Responsabilidad Social Empresarial del Sector Hotelero de la ciudad de Bucaramanga-Colombia. Estudios y Prospectivas en Turismo, 23(1), 23-39.

- Toro, D. (2006). El enfoque estratégico de la responsabilidad social corporativa: revisión de la literatura académica. Intangible Capital, 338-358.

- Torresano, M. (2012). Estudio de Responsabilidad Social de Empresas del Ecuador - 2012. (F. AVINA, Ed.) Ecuador: Noción Imprenta.

- Universo, D. e. (14 de 04 de 2017). Estado de derechos y justicia. Instituciones deciden qué es interés público, dice Viviana Paredes.

- Vives, A., \& Peinado - Vara, E. (2011). La Responsabilidad Social de la Empresa en América Latina. Washington: Cumputere. 